\title{
Rapid identification of dairy Propionibacterium species by restriction analysis of the insertion within the 23S rRNA gene
}

\author{
D. Fessler ${ }^{\mathrm{a}, \mathrm{b}}$, M.G. Casey ${ }^{\mathrm{a}}$, Z. Puhan ${ }^{\mathrm{b}}$ \\ ${ }^{a}$ Federal Dairy Research Institute, 3003 Bern, Switzerland \\ ${ }^{b}$ Laboratory of Dairy Science, Swiss Federal Institute of Technology, Zurich, Switzerland
}

(Received 17 February 1997; accepted 3 June 1997)

\begin{abstract}
An amplified sequence of approximately 350 bases from the 23S rRNA gene of each bacteria was used to identify 37 strains of dairy propionibacteria. After generation of the fragment by PCR (polymerase chain reaction), it was cut with the restriction endonuclease MspI. The resulting electrophoretic pattern showed a distinct profile for each of the Propionibacterium species. Purification of DNA was not necessary and the analyses could also be performed with crude bacterial extract. A collection of anaerobic bacteria commonly found in milk and milk products was also submitted to the analysis. None yielded the same pattern as propionibacteria. The analysis of the propionibacteria was compared to that performed by electrophoresis of the soluble cell-free protein extracts and that of classical microbiological tests. Our method gave identical results and was less time consuming (results could be obtained within a single day and were also easier to read) than both the classical tests and protein electrophoresis. (C) Inra/Elsevier, Paris.
\end{abstract}

\section{Propionibacterium / identification / PCR / restriction analysis / 23S rRNA}

Résumé - Identification rapide des espèces de bactéries propioniques laitières par analyse de restriction de l'insertion dans le gène de l'ARNr 23S. Une séquence amplifiée d'environ 350 bases du gène de l'ARNr $23 \mathrm{~S}$ de chaque bactérie a été utilisée pour l'identification de 37 souches de bactéries propioniques laitières. Le fragment a été amplifié par PCR (polymerase chain reaction), puis coupé par l'enzyme de restriction MspI. Le profil électrophorétique obtenu a été différent pour chaque espèce de bactérie propionique. Les analyses ont aussi été réalisées avec des extraits bruts de bactéries, sans purification préalable de l'ADN. Toute une série de bactéries anaérobies habituellement présenteś dans le lait a

\footnotetext{
* Correspondence and reprints
} 
également été soumise à cette analyse, sans que les profils obtenus aient correspondu à ceux des bactéries propioniques. Cette technique d'analyse a été comparée à celle de l'électrophorèse des extraits solubles des protéines et à celle des tests classiques de la microbiologie. Nos résultats étaient identiques à ceux des deux autres méthodes, mais ils ont été obtenus plus rapidement (en un seul jour) et sont de lecture plus facile. (C) Inra/Elsevier, Paris.

\section{Propionibacterium / identification / PCR / analyse de restriction / ARNr 23S}

\section{INTRODUCTION}

Propionibacteria were first isolated and described in 1909 by Orla-Jensen [16], and are today separated into eight species. Four were isolated from human skin: $P$. acnes, $P$. avidum, $P$. granulosum, and P. lymphophilum. The other four species consist of the dairy propionibacteria: $P$. freudenreichii, $P$. jensenii, $P$, thoenii, and $P$. acidipropionici. Propionibacteria are Gram-positive, non-motile, asporogenous, anaerobic to aerotolerant and generally catalase-positive pleomorphic rods [10]. Dairy propionibacteria are used in the manufacture of Emmental cheese with starters where they ferment lactic acid to propionic, acetic acid and $\mathrm{CO}_{2}$, which leads to the typical eye formation and flavor of this cheese. They can also cause cheese defects such as brown spots [5] and splitting $[13,15]$ in Emmental and in other types of Swiss cheese.

$P$ jensenii, $P$, thoenii and $P$, acidipropionici form a phylogenetical cluster, whereas $P$. freudenreichii is phylogenetically more remote [9]. The differentiation of the four dairy species, especially between $P$. thoenii and $P$. jensenii by classical bacteriological methods is apparently difficult. New methods have been proposed to facilitate their differentiation. Baer [2] and Baer and Ryba [3, 4] proposed identification systems based on protein electrophoresis, immunoblotting and serological studies. Riedel and Britz [19] and de Carvalho et al. [12] used ribotyping to differentiate propionibacteria based on the $16 \mathrm{~S}$ and the 23S rRNA. Riedel et al. [18] used restriction analysis of the 16S rRNA gene. Unfortunately, all of the methods are very time consuming.

Gram-positive bacteria with a high DNA G+C content are characterized by an insertion within their 23S rRNA genes [20]. Propionibacteria have a DNA G+C content of $65-67 \mathrm{~mol} \%$ [10] and the presence of the insertion was clearly confirmed [20]. In this paper a rapid and efficient method to classify dairy propionibacteria into their four species is described. It is based on the restriction analysis of a fragment of approximately 350 bases centered around the insertion.

\section{MATERIALS AND METHODS}

\subsection{Bacterial strains and culture conditions}

All 37 strains of propionibacteria were cultured anaerobically in YEL and on YELA (yeast extract lactate agar) for 5 to 7 days at $30^{\circ} \mathrm{C}$. YELA consists of $24 \mathrm{~mL} / \mathrm{L}$ sodium lactate $(50 \% \mathrm{v} / \mathrm{v}), 30 \mathrm{~g} / \mathrm{L}$ casein peptone, $30 \mathrm{~g} / \mathrm{L}$ yeast extract, and $15 \mathrm{~g} / \mathrm{L}$ agar; $\mathrm{pH}$ is adjusted to 6.8 .

The six strains of Clostridium were cultured in RCM broth [1] for 3 days at $37^{\circ} \mathrm{C}$. Enterococci strains were cultured for 2 days at $37^{\circ} \mathrm{C}$ in M17 [1] or on M17 agar with $0.05 \mathrm{~mol} / \mathrm{L}$ lactose. Lc. lactis strains were cultured in the 
same media as enterococci, but anaerobically for 2 days at $30^{\circ} \mathrm{C}$.

$S$. thermophilus was grown anaerobically for 2 days at $37^{\circ} \mathrm{C}$ in LS5 [8] or on LS5 agar with $0.05 \mathrm{~mol} / \mathrm{L}$ lactose.

L. delbrueckii was cultured anaerobically for 2 days at $37^{\circ} \mathrm{C}$ in MRS [1] or on MRS agar with $0.05 \mathrm{~mol} / \mathrm{L}$ lactose.

L. case $i$ strains were grown anaerobically for 2 days at $30^{\circ} \mathrm{C}$ in MRS with $0.05 \mathrm{~mol} / \mathrm{L}$ lactose and on FH-agar [14]. L. rhamnosus, $L$. zeae and $L$. plantarum were cultured in the same media as $L$, casei.

An overall view of the analyzed strains of bacteria is given in tables $I$ and $I I$.

\subsection{Preparation of crude DNA extracts}

\subsubsection{From liquid cultures}

$400 \mu \mathrm{L}$ of a liquid bacterial culture were centrifuged for $10 \mathrm{~min}$ at $9990 \mathrm{~g}$ in a Hettich Mikroliter centrifuge under sterile conditions. The sediment was suspended in $100 \mu \mathrm{L}$ sterile distilled water. The solution was boiled for $5 \mathrm{~min}$ and then centrifuged for $15 \mathrm{~min}$ at $9990 \mathrm{~g}$. $2 \mu \mathrm{L}$ of the supernatant were used for the PCRreaction.

\subsubsection{From colonies}

Bacterial material (one colony) was suspended in $100 \mu \mathrm{L}$ sterile distilled water. The solution was boiled for $5 \mathrm{~min}$ and then centrifuged for $15 \mathrm{~min}$ at $9990 \mathrm{~g} ; 2 \mu \mathrm{L}$ of the supernatant were used for the PCR-reaction.

\subsection{Polymerase chain reaction and restriction analysis}

Taq polymerase, PCR buffer and dNTP mix were purchased from Boehringer Mannheim and the primers from MWG-Biotech. The PCR-reaction was performed in $100 \mu \mathrm{L}$ containing $10 \mu \mathrm{L}$ PCR buffer $(10 \times$ concentrated $)$, $0.2 \mathrm{mmol} / \mathrm{L}$ dNTP $\operatorname{mix}(10 \mathrm{mmol} / \mathrm{L}$ each $), 0.1$ $\mu \mathrm{mol} / \mathrm{L}$ each of primers 5 -MADGCGTAGNCGAWGG-3 and 5 GTGWCGGTTTNBGGTA-3 [20], 2.5 u Taq DNA polymerase and $2 \mu \mathrm{L}$ crude bacterial extract.
PCR amplification was performed on a thermo-cycler from Inotech as follows: 25 cycles of $1 \mathrm{~min}$ at $95^{\circ} \mathrm{C}, 2 \mathrm{~min}$ at $52^{\circ} \mathrm{C}$, and 3 min at $72^{\circ} \mathrm{C}$ with a final step of $5 \mathrm{~min}$ at $72{ }^{\circ} \mathrm{C}$.

$1 \mu \mathrm{L}$ MspI (10 u/ $\mu \mathrm{L}$, Boehringer Mannheim) and $1 \mu \mathrm{L}$ BSA (bovine serum albumin, $20 \mathrm{mg} / \mathrm{mL}$, Boehringer Mannheim) were added to $20 \mu \mathrm{L}$ of the PCR amplification product and incubated for $2 \mathrm{~h}$ at $37^{\circ} \mathrm{C}$. Both restricted and untreated PCR amplification products were separated on a $4 \%$ agarose gel (NuSieve 3:1 agarose, FMC) and visualized under UV light in the presence of ethidium bromide buffer ( 10 $\mathrm{mmol} / \mathrm{L}$ sodium borate, $1 \mathrm{mg} / \mathrm{L}$ ethidium bromide, $\mathrm{pH} 8.0$ ). DNA size standard was 50-2000 bp ladder from BioRad with bands of $2000,1500,1000,700,500,400,300,200$, 100 and $50 \mathrm{bp}$.

\section{RESULTS AND DISCUSSION}

PCR with propionibacteria DNA and the chosen primers produced a single amplification product of approximately 350 bases confirming the results previously reported by Roller et al. [20]. Only Gram-positive bacteria with a high $\mathrm{G}+\mathrm{C}$ content possess a characteristic insertion of about 100 bases within their 23S rRNA genes [20].

Four endonucleases, which were compatible with PCR buffer and a high $\mathrm{G}+\mathrm{C}$ content, were tested: $C f o \mathrm{I}$ and $\mathrm{MvnI}$ digested the amplified fragment only partially (results not shown), whereas $M s p I$ and HpaII allowed differentiation of the four propionibacteria species. MspI and HpaII generated the same patterns and $M s p I$ was chosen for this study.

The patterns generated using the restriction endonuclease $M s p I$ were unique for each dairy Propionibacterium species. Figure $1 a$ shows the amplified fragment of approximately 350 bases of the four propionibacteria type strains and figure $1 \mathrm{~b}$ the patterns generated after restriction of this fragment with MspI. Comparison of the reference strains of propionibacteria in figure $1 b$ brings out clearly the diffe- 


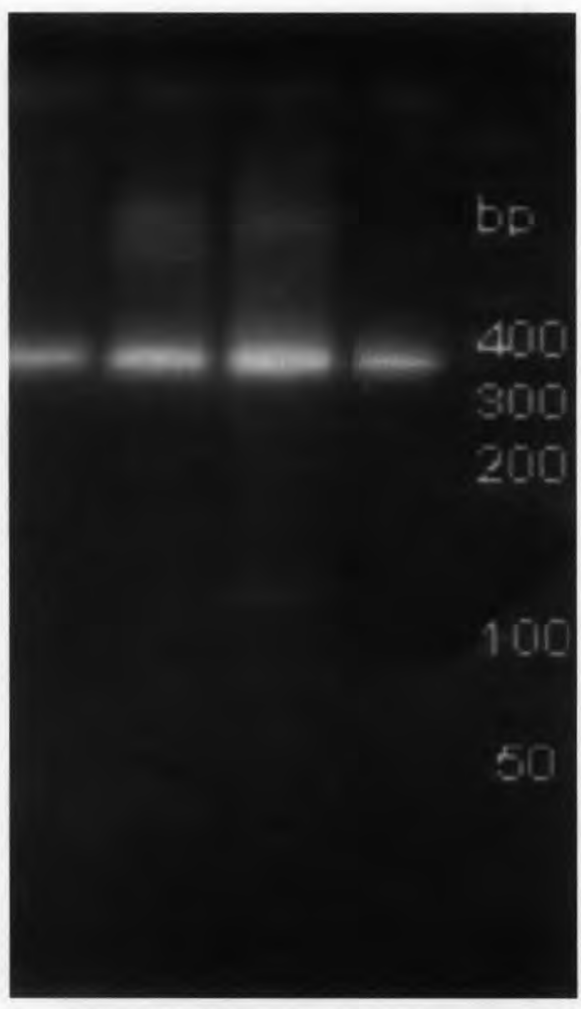

a.

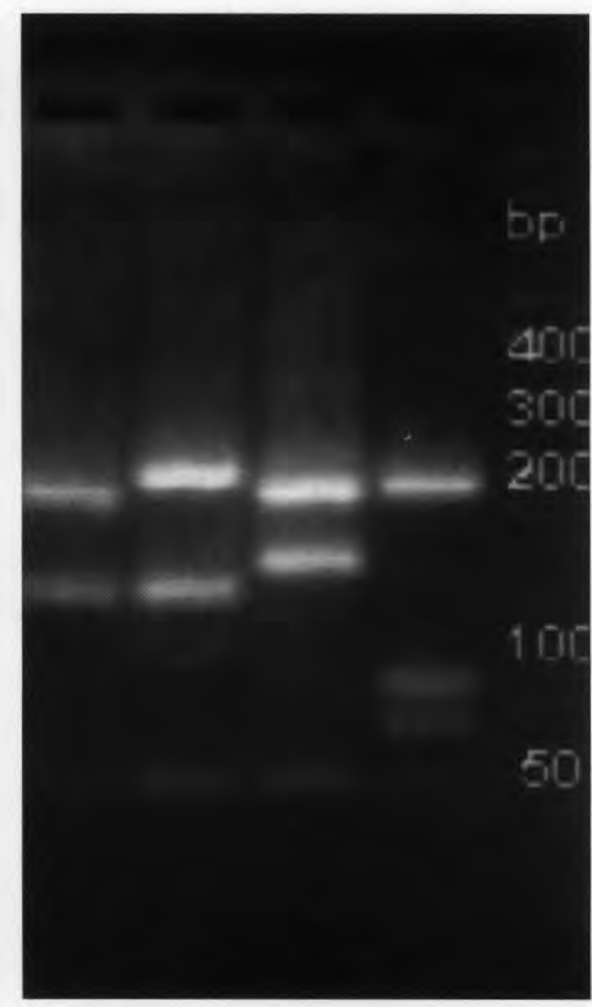

b.

Figure 1. a. Amplified fragments of the four Propionibacterium type strains. From left to right: 1) ATCC 25562 P. acidipropionici; 2) ATCC 4874 P. thoenii; 3) ATCC 4868 P. jensenii; 4) ATCC 6207 P. freudenreichii ssp. freudenreichii. b. Patterns generated after restriction with MspI of the amplified fragments of the four Propionibacterium type strains. From left to right: 1) ATCC 25562 P. acidipropionici; 2) ATCC $4874 P$. thoenii; 3) ATCC 4868 P. jensenii; 4) ATCC 6207 P. freudenreichii ssp. freudenreichii; 5) 50-2000 bp ladder.

Figure 1. a. Fragments amplifiés de quatre souches types de Propionibacterium. De gauche à droite : 1) ATCC 25562 P. acidipropionici; 2) ATCC $4874 P$. thoenii; 3) ATCC 4868 P. jensenii; 4) ATCC 6207 P. freudenreichii ssp. freudenreichii. b. Profils obtenus après restriction avec Msp. I du fragment amplifié de quatre souches types de Propionibacterium. De gauche à droite : 1) ATCC $25562 P$. acidipropionici; 2) ATCC $4874 P$. thoenii; 3) ATCC $4868 P$. jensenii; 4) ATCC 6207 P. freudenreichii ssp. freudenreichii; 5) 50-2000 bp ladder.

rences between the patterns of each species.

Restriction analysis of the fragment produced by $P$. freudenreichii gave three bands of approximately 195, 60 and 55 bp (figure 2). P. jensenii produced two bands of approximately 195 and 135 bp (figure 3), P. thoenii two bands of approximately 205 and 105 bp (figure 4), and P. acidipropionici two bands of approximately 190 and 120 bp (figure 5). Strains of all species produced an additional band of approximately 40 bp (figures $1 b-5$ ).

The strains from each species produced the same restriction profile (figures $2-5)$. Dairy propionibacteria could clearly 


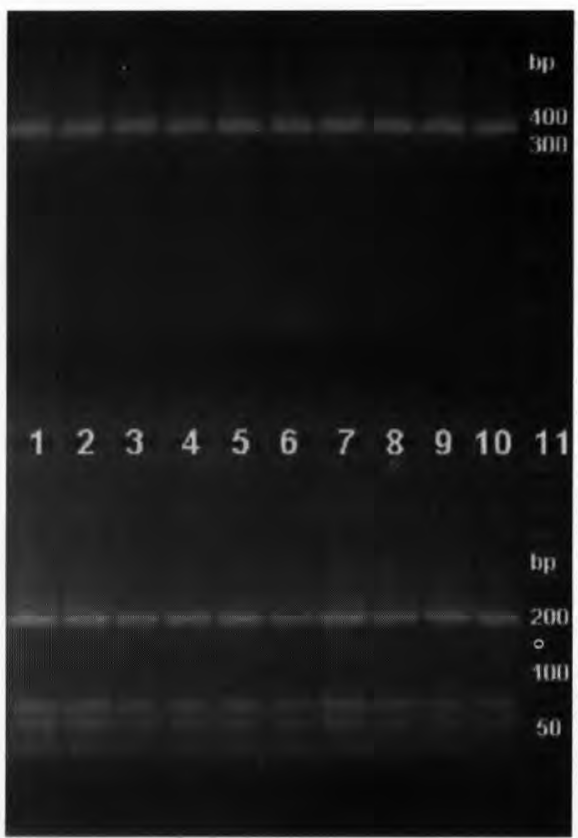

Figure 3. PCR analysis of $P$. jensenii, amplified fragments at the top, restriction patterns at the bottom. From left to right: 1) ATCC 4868; 2) DSM 20274; 3) DSM 20275; 4) DSM 20278; 5) DSM 20279; 6) AG 71.1; 7) BE28.1; 8) FR60.1; 9) SG 19.1 ; 10) ZS44.1;11) 50-2000 bp ladder.

Figure 3. Analyse de P. jensenii par PCR. En haut; fragments amplifiés ; en bas, fragments de restriction. De gauche à droite : 1) ATCC 4868 ; 2) DSM 20274 ; 3) DSM 20275 ; 4) DSM 20278 ; 5) DSM 20279 ; 6) AG 71.1 ; 7) BE28.1 ; 8) FR60.1 ; 9) SG19.1 ; 10) ZS44.1; 11) $50-2000$ bp ladder.

be classified into the four existing species $P$. freudenreichii, $P$. jensenii, $P$ thoenii, and $P$ acidipropionici. In order to facilitate comparison, it is recommended to use reference strains of every species, or at least of $P$. jensenii, $P$. thoenii, and $P$. acidipropionici, on each gel, since there is only a
Figure 2. PCR analysis of $P$. freudenreichii, amplified fragments at the top, restriction patterns at the bottom. From left to right: 1) ATCC 6207; 2) ATCC 9614; 3) DSM 20270; 4) P $1409 ; 5) \mathrm{P} 1410 ; 6$ ) $\mathrm{P} 1411$; 7) $\mathrm{P} 1412$; 8) $\mathrm{P}$ 1413; 9) P 1414; 10) P 111; 11) 50-2000 bp ladder.

Figure 2. Analyse de $P$. freudenreichii par PCR; en haut, fragments amplifiés; en bas, fragments de restriction. De gauche à droite : 1 ) ATCC 6207 ; 2) ATCC 9614 ; 3) DSM 20270 ; 4) $\mathrm{P} 1409$; 5) $\mathrm{P} 1410$; 6) $\mathrm{P} 1411$; 7) $\mathrm{P} 1412$; 8) P 1413 ; 9) P 1414 ; 10) P 111 ; 11) 50-2000 bp ladder.

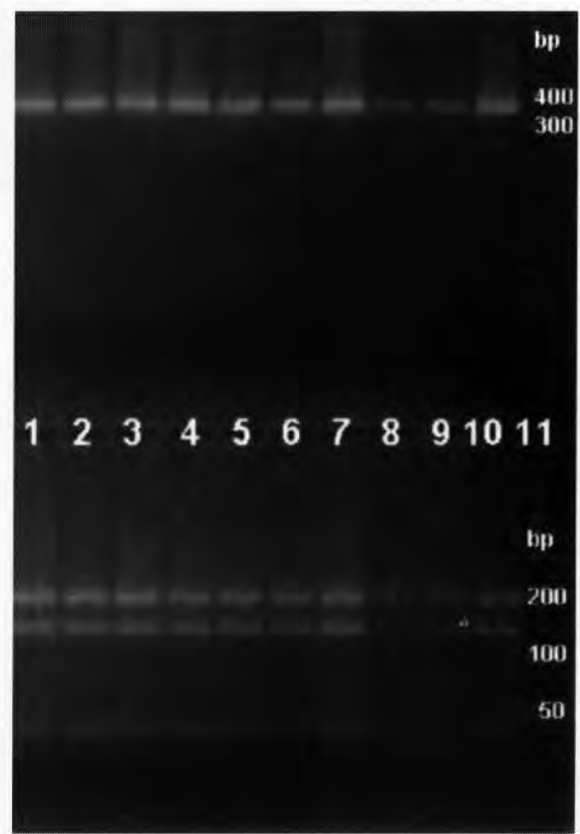

slight difference between $P$. acidipropionici and $P$. thoenii. The results in figures 1-5 were obtained with crude bacterial extracts from colonies. Identical results were obtained from extracts from liquid culture and with purified DNA (results not shown). 
Table I. Identification of propionibacteria isolates with different methods.

Tableau I. Identification d'isolats de bactéries propioniques par différentes méthodes.

\begin{tabular}{|c|c|c|c|c|c|}
\hline \multirow[t]{2}{*}{ Number } & \multirow[t]{2}{*}{ Strain } & \multirow[t]{2}{*}{ Source } & \multicolumn{3}{|c|}{ Identification } \\
\hline & & & Biochemical $^{1}$ & Proteins & PCR analysis \\
\hline $\mathrm{ATCC}^{2} 6207$ & P. freudenreichii ssp. freudenreichii $\mathrm{T}^{3}$ & $\mathrm{DSM}^{4}$ & - & P. freudenreichii & P. freudenreichii \\
\hline ATCC 9614 & P. freudenreichii ssp. shermanii $\mathrm{T}$ & DSM & - & P. freudenreichii & P. freudenreichii \\
\hline DSM 20270 & P. freudenreichii ssp. shermanii & DSM & - & P. freudenreichii & P. freudenreichii \\
\hline P 1409 & P. freudenreichii ssp. shermanii & $\mathrm{FAM}^{5}$ & - & P. freudenreichii & P. freudenreichii \\
\hline P 1410 & P. freudenreichii ssp. shermanii & FAM & - & P. freudenreichii & P. freudenreichii \\
\hline P 1411 & P. freudenreichii ssp. shermanii & FAM & - & P. freudenreichii & P. freudenreichii \\
\hline P 1412 & P. freudenreichii ssp. shermanii & FAM & - & P. freudenreichii & P. freudenreichii \\
\hline P 1413 & P. freudenreichii ssp. shermanii & FAM & - & P. freudenreichii & P. freudenreichii \\
\hline P 1414 & P. freudenreichii ssp. shermanii & FAM & - & P. freudenreichii & P. freudenreichii \\
\hline P 111 & P. freudenreichii ssp. shermanii & FAM & P. freudenreichii & P. freudenreichii & P. freudenreichii \\
\hline ATCC 4868 & P. jensenii $\mathrm{T}$ & DSM & - & P. jensenii & P. jensenii \\
\hline DSM 20274 & P. jensenii (zeae) & DSM & - & P. jensenii & P. jensenii \\
\hline DSM 20275 & P. thoenii (rubrum) & DSM & - & P. jensenii & P. jensenii \\
\hline DSM 20278 & P. jensenii & DSM & - & P. jensenii & $P$. jensenii \\
\hline DSM 20279 & $P$. jensenii (peterssonii) & DSM & - & P. jensenii & P. jensenii \\
\hline AG 71.1 & P. isolate & milk & P. jensenii & P. jensenii & P. jensenii \\
\hline BE 28.1 & P. isolate & milk & P. thoenii & P. jensenii & P. jensenii \\
\hline FR 60.1 & P. isolate & milk & P. jensenii & P. jensenii & P. jensenii \\
\hline SG 19.1 & P. isolate & milk & P. jensenii & P. jensenii & $P$. jensenii \\
\hline ZS 44.1 & P. isolate & milk & P. jensenii & $P$ jensenii & P. jensenii \\
\hline
\end{tabular}


Table I. Identification of propionibacteria isolates with different methods.

Tableau I. Identification d'isolats de bactéries propioniques par différentes méthodes.

\begin{tabular}{|c|c|c|c|c|c|}
\hline \multirow[t]{2}{*}{ Number } & \multirow[t]{2}{*}{ Strain } & \multirow[t]{2}{*}{ Source } & \multicolumn{3}{|c|}{ Identification } \\
\hline & & & Biochemical $^{1}$ & Proteins & PCR analysis \\
\hline ATCC 4874 & P. thoenii $\mathrm{T}$ & DSM & - & P. thoenii & P. thoenii \\
\hline DSM 20277 & P. thoenii & DSM & - & P. thoenii & P. thoenii \\
\hline AG 28.1 & P. isolate & milk & P. thoenii & P. thoenii & P. thoenii \\
\hline NW 36.1 & P. isolate & milk & P. thoenii & P. thoenii & P. thoenii \\
\hline ZS 22.1 & P. isolate & milk & P. thoenii & P. thoenii & P. thoenii \\
\hline 106.2 & P. isolate & milk & P. thoenii & P. thoenii & P. thoenii \\
\hline 204.1 & P. isolate & milk & P. jensenii & P. thoenii & P. thoenii \\
\hline 304.2 & P. isolate & milk & P. thoenii & P. thoenii & P. thoenii \\
\hline ATCC 25562 & P. acidipropionici $\mathrm{T}$ & DSM & - & P. acidipropionici & P. acidipropionici \\
\hline DSM 20272 & P. acidipropionici (pentosaceum) & DSM & - & P. acidipropionici & P. acidipropionici \\
\hline DSM 20273 & P. acidipropionici (arabinosum) & DSM & - & P. acidipropionici & P. acidipropionici \\
\hline AG 50.1 & P. isolate & milk & P. acidipropionici & P. acidipropionici & P. acidipropionici \\
\hline AG 66.1 & P. isolate & milk & P. acidipropionici & P. acidipropionici & P. acidipropionici \\
\hline BE 11.1 & P. isolate & milk & P. acidipropionici & P. acidipropionici & P. acidipropionici \\
\hline FR 55.2 & P. isolate & milk & P. acidipropionici & not identified & P. acidipropionici \\
\hline TG 17.2 & P. isolate & milk & P. acidipropionici & P. acidipropionici & P. acidipropionici \\
\hline ZS 44.2 & P. isolate & milk & P. acidipropionici & P. acidipropionici & P. acidipropionici \\
\hline
\end{tabular}

${ }^{1}$ According to fermentation pattern of carbohydrates by Cummins and Johnson [10]. ${ }^{2}$ American Type Culture Collection, Rockville, MD. ${ }^{3}$ Type strain. ${ }^{4}$ Deutsche Sammlung von Mikroorganismen und Zellkulturen $\mathrm{GmbH}$. ${ }^{5}$ Collection of Microorganisms of the Federal Dairy Research Institute, Switzerland.

${ }^{1}$ Selon la fermentation des hydrates de carbone, d'après Cummins et Johnson [10]. ${ }^{2}$ American Type Culture Collection, Rockville, MD. ${ }^{3}$ Souche type. ${ }^{4}$ Deutsche Sammlung von Mikroorganismen und Zellkulturen GmbH. ${ }^{5}$ Collection de microorganismes du Federal Dairy Research Institute, Suisse. 
Table II. Bacterial strains (except propionibacteria) analyzed in this study.

Tableau II. Souches bactériennes (autres que les bactéries propioniques) analysées dans cette étude.

\begin{tabular}{|c|c|c|}
\hline Number & Strain and biochemical identification ${ }^{1}$ & Source \\
\hline 2492 & L. delbrueckii ssp. bulgaricus $\mathrm{T}^{2}$ & $\mathrm{FAMb}^{3}$ \\
\hline 877 & L. delbrueckii ssp. lactis $\mathrm{T}$ & FAMb \\
\hline 144 & L. delbrueckii ssp. lactis & FAMb \\
\hline 145 & L. delbrueckii ssp. lactis & FAMb \\
\hline 169 & L. delbrueckii ssp. lactis & FAMb \\
\hline 170 & L. delbrueckii ssp. lactis & $\mathrm{FAMb}$ \\
\hline 278 & L. delbrueckii ssp. lactis & FAMb \\
\hline 286 & L. delbrueckii ssp. lactis & FAMb \\
\hline 933 & L. delbrueckii ssp. lactis & $\mathrm{FAMb}$ \\
\hline 1050 & L. delbrueckii ssp. lactis & FAMb \\
\hline 1051 & L. delbrueckii ssp. lactis & $\mathrm{FAMb}$ \\
\hline 1052 & L. delbrueckii ssp. lactis & $\mathrm{FAMb}$ \\
\hline 1086 & L. rhamnosus $\mathrm{T}$ & FAMb \\
\hline 1211 & L. rhamnosus & FAMb \\
\hline 1212 & L. rhamnosus & FAMb \\
\hline 1213 & L. rhamnosus & $\mathrm{FAMb}$ \\
\hline 1214 & L. rhamnosus & FAMb \\
\hline 1215 & L. rhamnosus & FAMb \\
\hline 1216 & L. rhamnosus & FAMb \\
\hline 1217 & L. rhamnosus & $\mathrm{FAMb}$ \\
\hline 1218 & L. rhamnosus & FAMb \\
\hline 1219 & L. rhamnosus & FAMb \\
\hline $1220 / 23$ & L. rhamnosus & FAMb \\
\hline 1116 & L. casei $\mathrm{T}$ & FAMb \\
\hline 1227 & L. casei & $\mathrm{FAMb}$ \\
\hline 1228 & L. casei & FAMb \\
\hline 1229 & L. casei & $\mathrm{FAMb}$ \\
\hline 1087 & L. plantarum $\mathrm{T}$ & $\mathrm{FAMb}$ \\
\hline 2051 & L. plantarum & FAMb \\
\hline 2053 & L. plantarum & FAMb \\
\hline 2057 & L. plantarum & FAMb \\
\hline 2062 & L. plantarum & FAMb \\
\hline 2063 & L. plantarum & FAMb \\
\hline 2064 & L. plantarum & FAMb \\
\hline 2065 & L. plantarum & FAMb \\
\hline 2066 & L. plantarum & FAMb \\
\hline 2069 & L. plantarum & FAMb \\
\hline 2071 & L. plantarum & FAMb \\
\hline 2075 & L. plantarum & $\mathrm{FAMb}$ \\
\hline 2076 & L. plantarum & FAMb \\
\hline 1084 & L. zeae $\mathrm{T}$ & FAMb \\
\hline
\end{tabular}


Table II. Bacterial strains (except propionibacteria) analyzed in this study.

Tableau II. Souches bactériennes (autres que les bactéries propioniques) analysées dans cette étude.

\begin{tabular}{|c|c|c|}
\hline Number & Strain and biochemical identification ${ }^{1}$ & Source \\
\hline 932 & S. thermophilus T & FAMb \\
\hline 879 & Enterococcus faecalis $\mathrm{T}$ & FAMb \\
\hline 2645 & Enterococcus faecium $\mathrm{T}$ & FAMb \\
\hline 3271 & Enterococcus sp. & FAMb \\
\hline 3272 & Enterococcus sp. & FAMb \\
\hline 3273 & Enterococcus sp. & $\mathrm{FAMb}$ \\
\hline 3274 & Enterococcus sp. & FAMb \\
\hline 3275 & Enterococcus sp. & FAMb \\
\hline 3276 & Enterococcus sp. & $\mathrm{FAMb}$ \\
\hline 3277 & Enterococcus sp. & $\mathrm{FAMb}$ \\
\hline 2493 & Lactococcus lactis $\mathrm{T}$ & FAMb \\
\hline 3009 & Lactococcus lactis & FAMb \\
\hline 3010 & Lactococcus lactis & FAMb \\
\hline 3011 & Lactococcus lactis & FAMb \\
\hline 3013 & Lactococcus lactis & FAMb \\
\hline 3018 & Lactococcus lactis & $\mathrm{FAMb}$ \\
\hline 3233 & Lactococcus lactis & FAMb \\
\hline 552 & C. butyricum $\mathrm{T}$ & $\mathrm{DSM}^{4}$ \\
\hline 1322 & C. butyricum & FAM $^{5}$ \\
\hline 795 & C. sporogenes $\mathrm{T}$ & DSM \\
\hline 1755 & C. sporogenes $\mathrm{T}$ & FAM \\
\hline 2637 & C. tyrobutyricum $\mathrm{T}$ & DSM \\
\hline 608 & C. tyrobutyricum & $\mathrm{CNRZ}^{6}$ \\
\hline
\end{tabular}

'D'après Cummins et Johnson [10]. ${ }^{2}$ Souche type. ${ }^{3}$ Biochimie, Federal Dairy Research Institute, Suisse. ${ }^{4}$ Deutsche Sammlung von Mikroorganismen und Zellkulturen $\mathrm{GmbH}$. ${ }^{5}$ Collection de microorganismes du Federal Dairy Research Institute, Suisse. ${ }^{6}$ Centre national de recherches zootechniques, Jouy-en-Josas, France.

${ }^{1}$ According to Cummins and Johnson [10]. ${ }^{2}$ Type strain. ${ }^{3}$ Biochemistry, Federal Dairy Research Institute, Switzerland. ${ }^{4}$ Deutsche Sammlung von Mikroorganismen und Zellkulturen $\mathrm{GmbH}$. ${ }^{5}$ Collection of Microorganisms of the Federal Dairy Research Institute, Switzerland. ${ }^{6}$ Centre national de recherches zootechniques, Jouy-enJosas, France.

The classification of propionibacteria strains with our method is in agreement with that obtained from protein electrophoresis patterns (table I). Strain FR 55.2, which could not be clearly identified by protein electrophoresis, was classified as $P$. acidipropionici by restriction analysis as well as by biochemical methods.
For $P$. freudenreichii and P. acidipropionici the results obtained with PCR analysis are also in agreement with those obtained by classical biochemical methods. For $P$. jensenii the correspondence between the two methods is $90 \%$ and for P. thoenii $87.5 \%$ (table I). However, it is known, that the identification of dairy Propionibacterium species with classical 


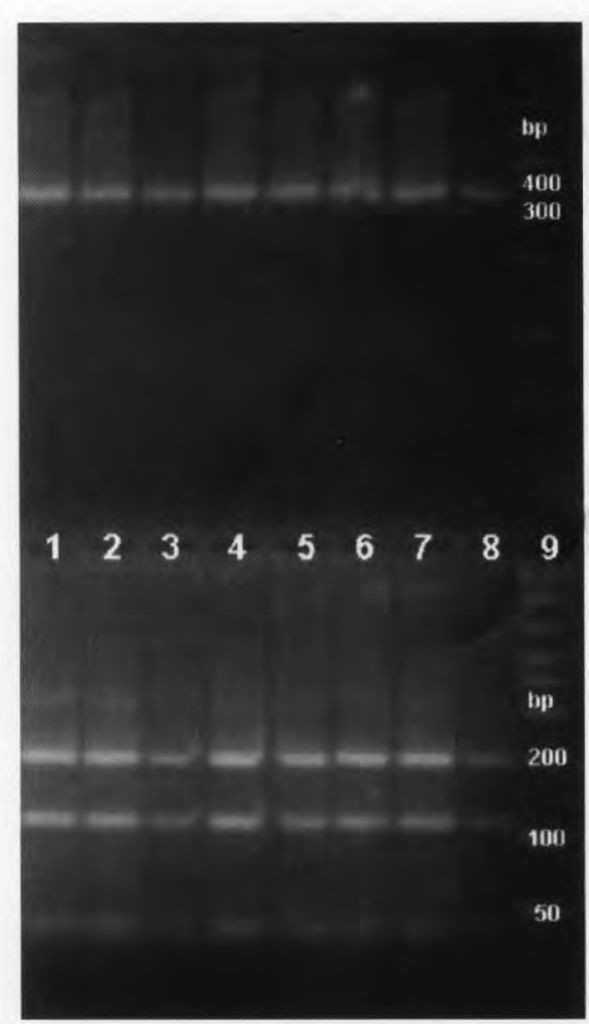

Figure 5. PCR analysis of $P$. acidipropionici; amplified fragments at the top; restriction patterns at the bottom. From left to right: 1) ATCC 25562 ; 2) DSM 20272; 3) DSM 20273; 4) AG 50.1 ; 5) AG 66.1; 6) BE 11.1 ; 7) FR 55.2; 8) TG 17.2; 9) ZS 44.2; 10) 50-2000 bp ladder.

Figure 5. Analyse de $P$. acidipropionici par PCR. En haut, fragments amplifiés ; en bas, fragments de restriction. De gauche à droite : 1) ATCC 25562 ; 2) DSM 20272 ; 3) DSM 20273 ; 4) AG 50.1 ; 5) AG 66.1 ; 6) BE 11.1 ; 7) FR 55.2 ; 8) TG 17.2 ; 9) ZS 44.2; 10) 50-2000 bp ladder.

methods may be ambiguous $[6,11]$. The greatest problem seems to be the distinction between $P$. jensenii and $P$. thoenii. Both of these species could clearly be differentiated with the method described here and the earlier $P$. rubrum was classified
Figure 4. PCR analysis of $P$ thoenii, amplified fragments at the top, restriction patterns at the bottom. From left to right: 1) ATCC $4874 ; 2$ ) DSM 20277; 3) AG 28.1; 4) NW 36.1; 5) ZS 22.1 ; 6) 106.2; 7) 204.1; 8) 304.2; 9) 50-2000 bp ladder.

Figure 4. Analyse de $P$. thoenii par PCR. En haut, fragments amplifiés; en bas, fragments de restriction. De gauche à droite : 1) ATCC 4874; 2) DSM 20277 ; 3) AG 28.1 ; 4) NW 36.1 ; 5) ZS 22.1 ; 6) 106.2 ; 7) 204.1 ; 8) 304.2; 9) 50-2000 bp ladder.

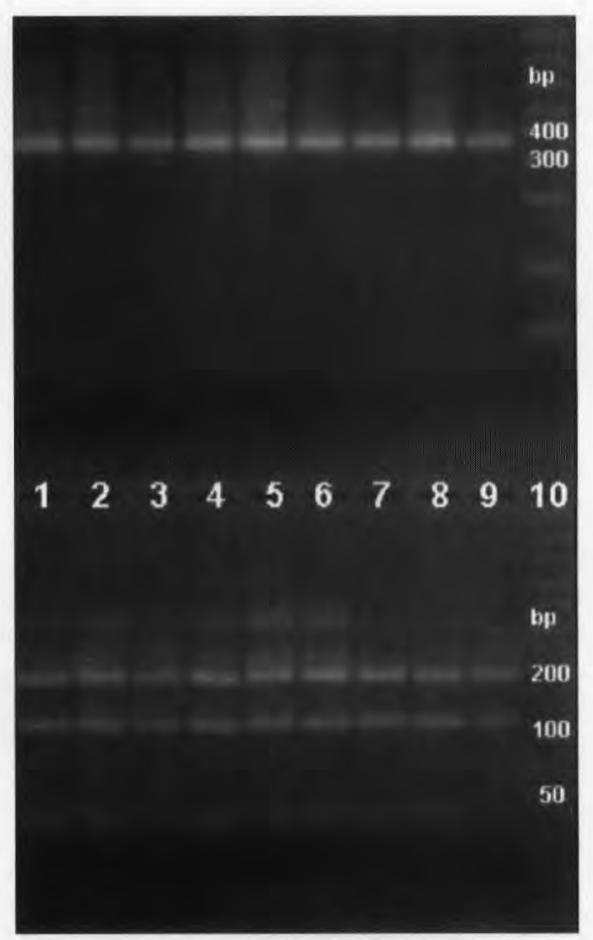

under $P$. jensenii (figure 3 ). This finding is in agreement with the classification of $P$. rubrum as $P$. jensenii by Britz and Steyn [7], Britz and Riedel [6], de Carvalho et al. [11], Riedel et al. [18] and recently by Riedel and Britz [19]. 
Figure 6. PCR analysis of other bacteria; amplified fragments at the top; restriction patterns at the bottom. From left to right: 1) 50-2000 bp ladder; 2) 932; 3) 1084 ; 4) 2076; 5) 2075 ; 6) 2071 ; 7) 2069 ; 8) 2066 ; 9) 2065 ; 10) 2064 ; 11) 2063 ; 12) 2062 ; 13) 2057 ; 14) 2053; 15) 2051; 16) 1087; 17) ATCC 25562 P. acidipropionici $\mathrm{T} ; 18$ ) ATCC 4874 P. thoenii T; 19) ATCC 4868 P. jensenii $\mathrm{T}$; 20) ATCC 6207 P. freudenreichii ssp. freudenreichii $\mathrm{T}$; 21) 50-2000 bp ladder.

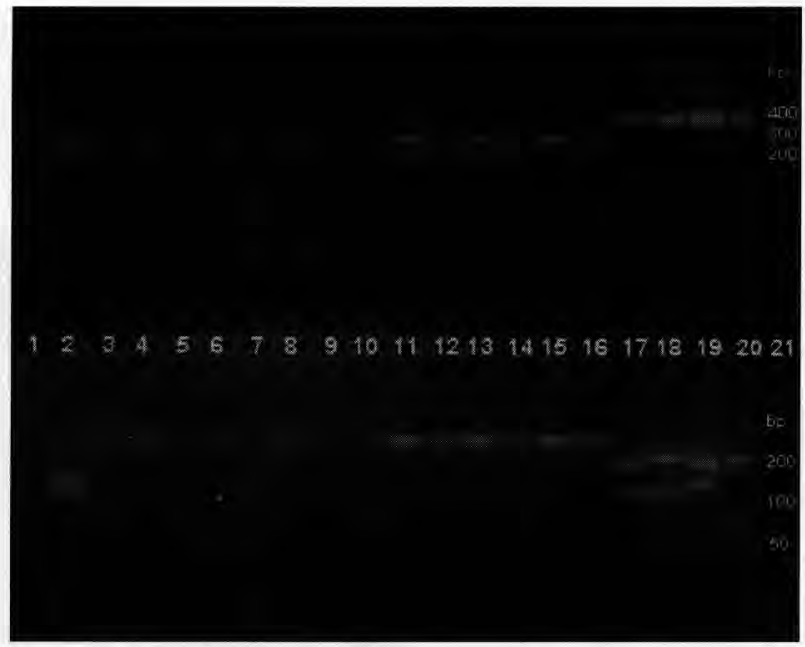

Figure 6. Analyse d'autres bactéries par PCR. En haut, fragments amplifiés ; en bas, fragments de restriction. De gauche à droite : 1) 50 -2000 bp ladder ; 2) 932 ; 3) 1084 ;4) 2076 ; 5) 2075 ; 6) 2071 ; 7) 2069 ; 8) 2066 ; 9) 2065 ; 10) 2064 ; 11) 2063 ; 12) 2062 ; 13) 2057 ; 14) 2053 ; 15) 2051 ; 16) 1087 ; 17) ATCC 25562 P. acidipropionici T ; 18) ATCC 4874 P. thoenii T ; 19) ATCC $4868 P$. jensenii T ; 20) ATCC 6207 P. freudenreichii ssp. freudenreichii T ; 21) 50-2000 bp ladder.

Figure 7. PCR analysis of other bacteria; amplified fragments at the top; restriction patterns at the bottom. From left to right: 1) 50-2000 bp ladder; 2) 3233; 3) 3018; 4) 3013; 5) 3011; 6) 3010 ; 7) 3009 ; 8) 2493; 9) 3277 ; 10) 3276 ; 11) 3275 ; 12) 3274; 13) 3273 ; 14) 3272; 15) 3271; 16) 2645 ; 17) 879 ; 18) ATCC 25562 P. acidipropionici T; 19) ATCC 4874 P. thoenii T; 20) ATCC 4868 P. jensenii T; 21) ATCC 6207 P. freudenreichii ssp. freudenreichii T; 22) 50-2000 bp ladder.

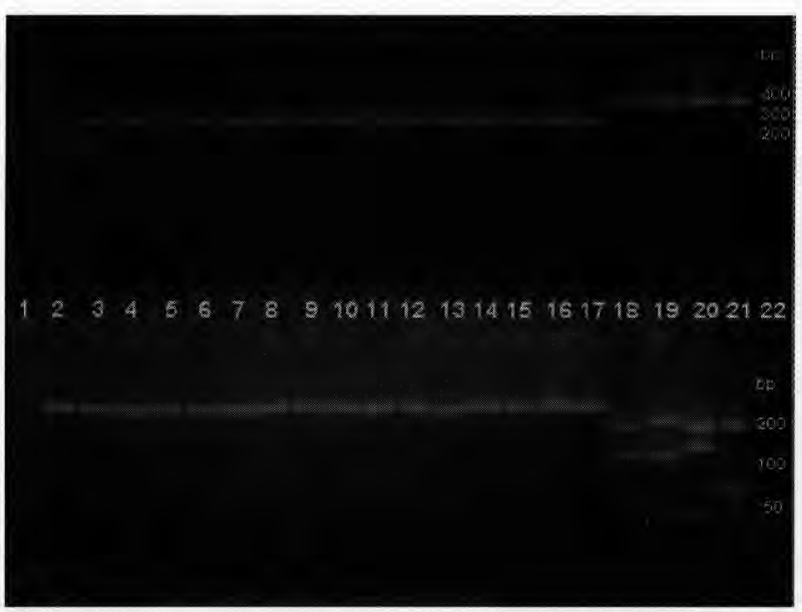

Figure 7. Analyse d'autres bactéries par PCR. En haut, fragments amplifiés ; en bas, fragments de restriction. De gauche à droite : 1) 50-2000 bp ladder ; 2) 3233 ; 3) 3018 ; 4) 3013 ; 5) 3011 ; 6) 3010 ; 7) 3009 ; 8) 2493 ; 9) 3277 ; 10) 3276 ; 11) 3275 ; 12) 3274 ; 13) 3273 ; 14) 3272 ; 15) 3271 ; 16) 2645 ; 17) 879 ; 18) ATCC 25562 P. acidipropionici $\mathrm{T}$; 19) ATCC $4874 P$. thoenii T ; 20) ATCC $4868 P$. jensenii T ; 21) ATCC 6207 P. freudenreichii $\mathrm{ssp}$. freudenreichii $\mathrm{T}$; 22) $50-2000 \mathrm{bp}$ ladder. 
Figure 8. PCR analysis of other bacteria; amplified fragments at the top; restriction patterns at the bottom. From left to right: 1) 50-2000 bp ladder; 2) 1213; 3) 1212; 4) 1211; 5) 1086; 6) 1052; 7) 1051 ; 8) 1050 ; 9) 933 ; 10) 286; 11) 278 ; 12) 170;13) 169; 14) 145 ; 15) 144; 16) 877; 17) 2494; 18) ATCC 25562 P. acidipropionici T; 19) ATCC 4874 P. thoenii T; 20) ATCC 4868 P. jensenii T; 21) ATCC 6207 P freudenreichii ssp. freudenreichii T; 22) 50-2000 bp ladder.

Figure 8. Analyse d'autres bactéries par PCR. En haut, fragments amplifiés; en bas, fragments de restriction. De gauche à droite : 1) 50-2000 bp ladder ;2) 1213 ; 3) 1212 ; 4) 1211 ; 5) 1086 ; 6) 1052 ; 7) 1051 ; 8) 1050 ; 9) 933 ; 10) 286 ; 11) 278 ; 12) 170 ; 13) 169 ; 14) 145 ; 15) 144 ; 16) 877 ; 17) 2494 ; 18) ATCC $25562 P$. acidipropionici $\mathrm{T}$; 19) ATCC 4874 P. thoenii $\mathrm{T} ; 20)$ ATCC $4868 P$. jensenii $\mathrm{T} ; 21$ ) ATCC 6207 P. freudenreichii ssp. freudenreichii $\mathrm{T}$; 22) 50-2000 bp ladder.

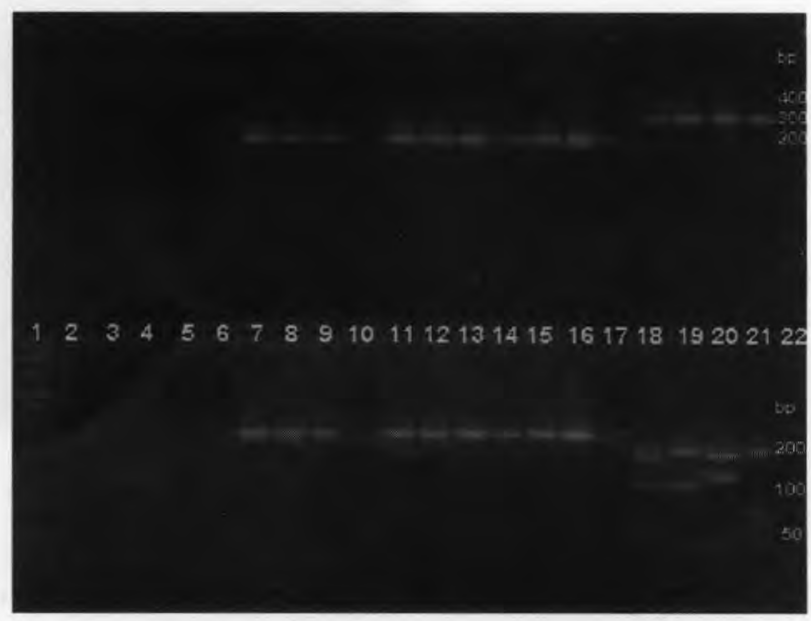

Figure 9. PCR analysis of other bacteria; amplified fragments at the top; restriction patterns at the bottom. From left to right: 1) 50-2000 bp ladder; 2) 608; 3) 2637; 4) 1755 ; 5) 795; 6) 1322; 7) 552; 8) 1229 ; 9) 1228 ; 10) 1227 ; 11) 1116; 12) 1220/23; 13) 1219; 14) 1218; 15) 1217; 16) 1216;17) 1215 ; 18) 1214; 19) ATCC 25562 P. acidipropionici T; 20) ATCC 4874 P. thoenii T; 21) ATCC 4868 P. jensenii T; 22) ATCC 6207 P. freudenreichii ssp. freudenreichii T; 23) 50-2000 bp ladder.

Figure 9. Analyse d'autres bactéries par PCR. En haut, fragments amplifiés ; en bas, fragments de restriction. De gauche à droite : 1) 50-2000 bp ladder ; 2) 608 ; 3) 2637 ; 4) 1755 ; 5) 795 ; 6) 1322 ;7) 552 ;8) 1229 ;9) 1228 ; 10) 1227 ; 11) 1116 ; 12) $1220 / 23$; 13) 1219 ; 14) 1218 ; 15) 1217 ; 16) 1216 ; 17) 1215 ; 18) 1214 ; 19) ATCC $25562 P$. acidipropionici $\mathrm{T} ; 20$ ) ATCC 4874 P. thoenii T ; 21) ATCC $4868 P$. jensenii $\mathrm{T} ; 22$ ) ATCC 6207 P. freudenreichii ssp. freudenreichii $\mathrm{T}$; 23) 50-2000 bp ladder.

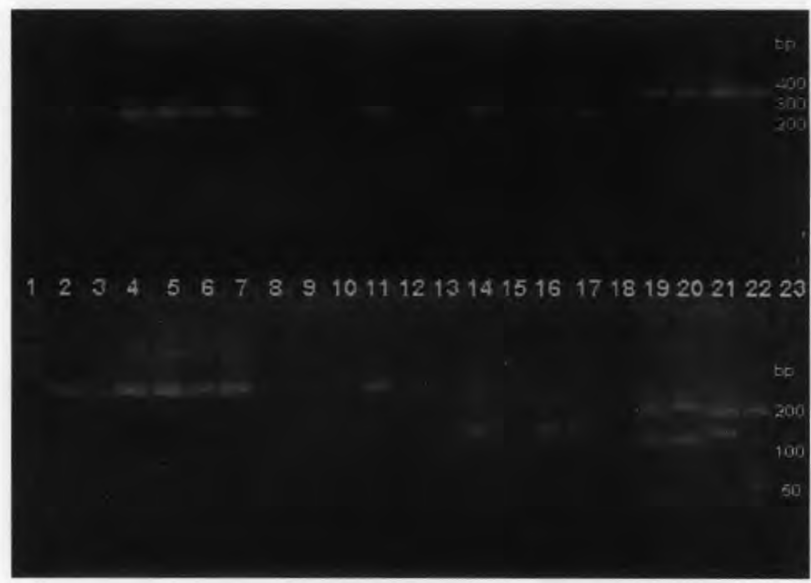


Protein electrophoresis of propionibacteria yields a more detailed image of the analyzed strains. It produces far more bands than the two or three obtained by restriction analysis. Based on protein profiles propionibacteria strains can not only be classified into their species, but can also be grouped into clusters within the species [17]. However, when it is not necessary to obtain a more detailed analysis of a strain than its species, restriction analysis on the 23S rRNA gene is much faster and the patterns are simpler to read than protein profiles.

PCR with the chosen primers was carried out with different genera (table $I I$ ) of anaerobic bacteria naturally occurring in milk or dairy products, such as lactobacilli, enterococci, clostridia, streptococci, and lactococci. Figures 6-9 show that only propionibacteria produced a fragment of approximately 350 bases and gave a unique pattern after restriction enzyme analysis with $M s p I$. Many of the bacteria encountered in milk would be expected to produce a smaller fragment than propionibacteria. Indeed most of the other bacteria generated a smaller and weaker fragment of about 250 bases. This fragment was not or only partially cut with the restriction endonuclease $M s p I$. The genus of propionibacteria was clearly distinguishable from the other tested bacteria even without cutting the fragment of about 350 bases (figures 6-9). Approximately 100 unidentified strains isolated from milk, growing on lactate, and morphologically as well as microscopically similar to propionibacteria were analyzed. PAGE (polyacrylamide gel electrophoresis) of their proteins demonstrated that they were not propionibacteria. None of these bacteria showed one of the four typical profiles of propionibacteria (results not shown).

The method described here is a rapid, simple and reliable technique to distinguish between the Propionibacterium species. It yields reproducible results and can be performed with liquid cultures or bacterial material from colonies, as there is no need to purify DNA. A large number of samples, approximately 40 , depending on the thermocycler and the electrophoresis apparatus used, can be analyzed within a single day. This method could in the future help to distinguish other Gram-positive bacteria with a high $\mathrm{G}+\mathrm{C}$ content.

\section{ACKNOWLEDGMENTS}

We thank N. Loosli, E. Wagner and Dr. J. Jimeno for providing us with bacterial cultures.

\section{REFERENCES}

[1] Atlas R.M., in: Parks L.C., (Ed), Handbook of microbiological media, CRC Press, Florida, 1993, pp. $532,621,765$

[2] Baer A., Identification and differentiation of propionibacteria by electrophoresis of their proteins, Milchwissensehaft 42 (1987) 431-433.

[3] Baer A., Ryba I., Identification or propionibacteria and streptococci by immunoblotting, Milchwissenschaft 46 (1991) 292-294.

[4] Baer A., Ryba I., Serological identification of propionibacteria in milk and cheese samples, Int. Dairy J. 2 (1992) 299-310.

[5] Baer A., Ryba I., Grand M,, Ursachen der Entstehung von braunen Tupfen im Käse, Schweiz. Milchw. Forsch. 22 (1993) 3-7.

[6] Britz T.J., Riedel K.H.J., A numerical taxonomic study of Propionibacterium strains from dairy sources, J. Appl. Bacteriol. 71 (1991) 407-416.

[7] Britz T.J., Steyn P.L.. Comparative studies on propionic acid bacteria, Phytophylactica 12 (1980) 89-103.

[8] Casey M.G., Meyer J., Presence of X-prolyldipeptidyl-peptidase in lactic acid bacteria, J. Dairy Sci. 68 (1985) 3212-3215.

[9] Charfreitag O., Stackebrandt E., Inter- and intrageneric relationships of the genus Propionibacterium as determinated by $16 \mathrm{~S}$ rRNA sequences, J. Gen. Microbiol. 135 (1989) 2065-2070.

[10] Cummins C.S., Johnson J.L., Genus I. Propionibacterium Orla-Jensen 1909. in: Sneath P.H.A., Mair N.S., Sharpe M.E., Holt J.G. (Eds.), Bergey's Manual of Systematic Bacteriology Vol. 2. The Williams \& Wilkins Co, Baltimore, 1986, pp. 1346-1353. 
[11] De Carvalho A.F., Systématique des bactéries propioniques laitières: classification, nomenclature et identification, Dissertation 9429, 69, ENSA de Rennes, 1994.

[12] De Carvalho A.F., Gautier M., Grimont P.A.D., Identification of dairy Propionibacterium species by rRNA gene restriction patterns, Res. Microbiol. 145 (1994) 667-676.

[13] Hettinga D.H., Reinbold G.W., Vedamuthu E.R., The split defect of Swiss cheese. I. Effect of strain of Propionibacterium and wrapping material, J. Milk Food Technol. 37 (1974) 322-328.

[14] Isolini D., Grand M., Glättli H., Selektivmedien zum Nachweis von obligat und fakultativ heterofermentativen Laktobazillen, Schweiz Milchw. Forschung 19 (1990) 57-59.

[15] Park H.S., Reinbold G.W., Hammond E.G., Role of propionibacteria in split defect of Swiss cheese, J. Dairy Sci. 50 (1967) $820-823$.
[16] Orla-Jensen S., Die Hauptlinien des natürlichen Bakteriensystems, Zent. Bakteriol., Parasitenk. Infektionskr. Abteilung 2 (1909) 305-346.

[17] Riedel K.H.J., Britz T.J., Differentiation of 'classical' Propionibacterium species by numerical analysis of electrophoretic protein profiles, Syst. Appl. Microbiol. 15 (1992) 567-572.

[18] Riedel K.H.J., Wingfield B.D., Britz T.J., Justification of the 'classical' Priopionibacterium species concept by restriction analysis of the 16S ribosomal RNA genes, Syst. Appl. Microbiol. 17 (1994) 536-542.

[19] Riedel K.H.J., Britz T.J., Justification of the 'classical' Propionibacterium species concept by ribotyping, Syst. Appl. Microbiol. 19 (1996) 370-380.

[20] Roller C., Ludwig W., Schleifer K.H., Grampositive bacteria with a high DNA $\mathrm{G}+\mathrm{C}$ content are characterized by a common insertion within their 23S rRNA genes, J. Gen. Microbiol. 138 (1992) 1167-1175. 